\title{
Deeply Virtual Compton Scattering off the Neutron with Spectator Proton Tagging in Hall A at Jefferson Laboratory
}

Eric Fuchey ${ }^{* \dagger}$

University of Connecticut

E-mail: efuchey@jlab.org

\begin{abstract}
Generalized Parton Distributions (GPDs) provide a three-dimensional parametrization of the quarks and gluons inside the nucleon, which provides a particularly valuable insight on the orbital angular momentum of quarks and gluons. GPDs may be determined with measurement of exclusive electroproduction processes such as Deeply Virtual Compton Scattering (DVCS) $e N \rightarrow e N \gamma$. In particular, measurement on the neutron, combined with measurements on the proton, provides a particularly interesting combination of observables which give a strong constraint on the orbital angular momentum of partons. We propose to measure the Deeply Virtual Compton Scattering (DVCS) on the neutron, using a deuterium target, and with spectator proton tagging. Such a technique allows to unambiguously identify the neutron-DVCS events from proton and coherent deuterium DVCS events, and to apply corrections to the nuclear effect of the neutron in the deuteron. We describe here this experiment, its assets, and projections will be discussed.
\end{abstract}

23rd International Spin Physics Symposium - SPIN2018 -

10-14 September, 2018

Ferrara, Italy

*Speaker.

${ }^{\dagger}$ on behalf of nDVCS SBS collaboration 


\section{Introduction: physics motivations}

Generalized parton distributions (GPDs) are a new theoretical tool, developed in the late 90s, which link form factors and parton distributions. They offer correlation information between the transverse location and the longitudinal momentum of partons and can access the contribution of the orbital angular momentum of quarks (and gluons) to the nucleon spin [1, 2, 3].

Deeply Virtual Compton Scattering (DVCS) on the nucleon is the simplest hard exclusive process involving GPDs, for which can be seen a leading order diagram in Fig. 1.

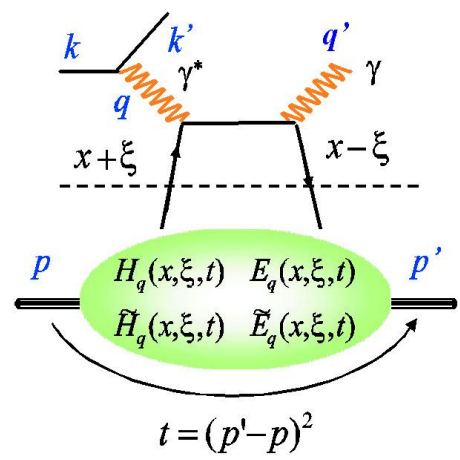

Figure 1: The handbag diagram for DVCS. An incident lepton of four-momentum $k$ generates a virtual photon of four-momentum $q=k-k^{\prime}$. The momentum four-vector of the scattered photon is $q^{\prime}$. The initial and final momentum four-vectors of the nucleon are $p$ and $p^{\prime}$ resulting in a total momentum transfer $t=$ $\left(p^{\prime}-p\right)^{2}=\left(q-q^{\prime}\right)^{2}$. Additional $\left(e, e^{\prime}\right)$ invariants are $y=q \cdot p /(k \cdot p), W^{2}=(q+p)^{2}, Q^{2}=-q^{2}$, and $x_{B}=Q^{2} /(2 q \cdot p)$. The GPD skewness invariant is $\xi=x_{B} /\left(2-x_{B}\right)$.

The amplitude to remove and restore the parton inside the nucleon is described, at the leading order in $1 / Q$, in terms of four GPDs $H, \tilde{H}, E$ and $\tilde{E}$ depending of the three variables $x, \xi$ and the momentum transfer $t$.

Experimentally DVCS interferes with the Bethe-Heitler $(\mathrm{BH})$ process where the real photon is radiated by the incident or the scattered lepton. Since the $\mathrm{BH}$ is purely real and fully calculable with the known form factors, this means that at leading order, the DVCS amplitude may be accessed directly by measuring the beam spin asymmetry of $e n \rightarrow e n \gamma$, which is proportional to the DVCS$\mathrm{BH}$ interference term, and appears as a $\sin \phi_{\gamma \gamma}$ modulation.

To investigate neutron structure via electron scattering, a deuterium target frequently serves as a quasi-free neutron target because of the weak binding energy between the proton and the neutron inside the deuteron. Within the impulse approximation (IA), where only one nucleon is active and participates in the absorption and emission of the photon (the other nucleon being a spectator), the electroproduction of photons on a deuterium target may be decomposed into elastic (d-DVCS) and quasi-elastic (p-DVCS and n-DVCS) contributions:

$$
\mathrm{D}\left(\vec{e}, e^{\prime} \gamma\right) X=d\left(\vec{e}, e^{\prime} \gamma\right) d+n\left(\vec{e}, e^{\prime} \gamma\right) n+p\left(\vec{e}, e^{\prime} \gamma\right) p+\ldots
$$

The neutron helicity dependent cross section is mostly sensitive to $E( \pm \xi, \xi, t)$, the least constrained GPD [4]. The knowledge of $E$ is essential because it enters on equal footing with $H$ in Ji's sum rule leading to the total angular momentum carried by quarks in the nucleon [3]. In addition to providing different GPDs combinations, the neutron experiments have naturally a different flavor 
sensitivity to GPDs than the proton experiments and they appear as a mandatory step towards a better knowledge of the partonic structure of the nucleon. Figure 2 is a beautiful illustration of this complementarity between the neutron and the proton experiments.

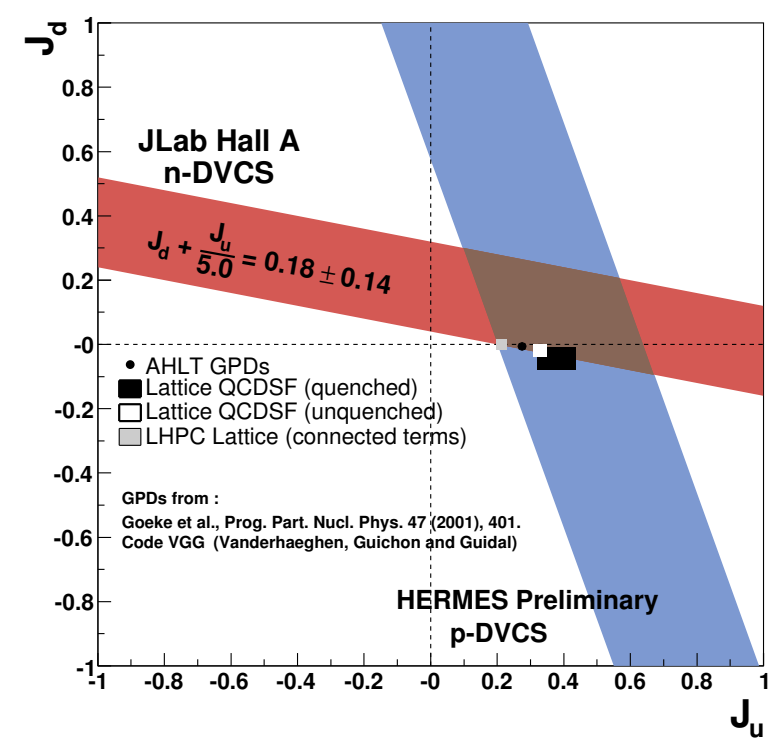

Figure 2: A model-dependent extraction of the contributions $J_{u}$ and $J_{d}$ of up- and down-quarks, respectively to the proton spin [4].

\section{Experiment}

\subsection{Overview}

We propose to measure the exclusive photon and neutral pion electroproduction on deuterium, with identification of the spectator proton $D\left(e, e^{\prime} \gamma p_{\text {spec }}\right) n$ and $D\left(e, e^{\prime} \pi^{0} p_{\text {spec }}\right) n$, in the valence region $(x>0.1)$ and deep inelastic regime $\left(Q^{2}>1 \mathrm{GeV}^{2}, W^{2}>2 \mathrm{GeV}^{2}\right)$.

The key to this experimental technique is to measure the very low-energy outgoing spectator proton, in triple coincidence with the deeply inelastically scattered electron and the high energy photon (or pair of high energy photons in the $\pi^{0}$ case).

The analysis of double coincidence events $D\left(e, e^{\prime} \gamma\right) n$, together with $H\left(e, e^{\prime} \gamma\right) p$, to extract Deeply Virtual Compton Scattering (DVCS) cross sections on the neutron (by subtraction of DVCS on hydrogen from the total exclusive photon production off deuterium) has already proved successful [4], albeit with large systematic uncertainties on the estimation of $n\left(e, e^{\prime} \gamma\right) n$.

The identification of the DVCS events off the neutron can be obtained with the measurement of the recoil neutron, as it has been proposed by [5], or with the identification of the spectator proton, which is the measurement we propose in this document, and which has also been proposed by ALERT [6]. The latter technique has the advantage over the former that the spectator proton momentum and vertex can be measured with significantly better precision (if not with significantly better efficiency) than the recoil neutron. Plus, it gives an handle on the Fermi momentum of the struck neutron. Both effects greatly reduce the systematic uncertainties. 
Our experiment proposes to perform our measurement using the Tagged Deep Inelastic Scattering experiment (TDIS) [7] in Hall A. The TDIS experiment will employ the Super BigBite Spectrometer (SBS) to detect the scattered electrons, in time and vertex coincidence with low momentum proton(s) measured in a low radiation length radial modular Time Projection Chamber (mTPC). Our measurement will use the exact same setup, with the addition of a calorimeter to detect the high energy photon. A representation of this setup is shown on Fig. 3. As for the TDIS

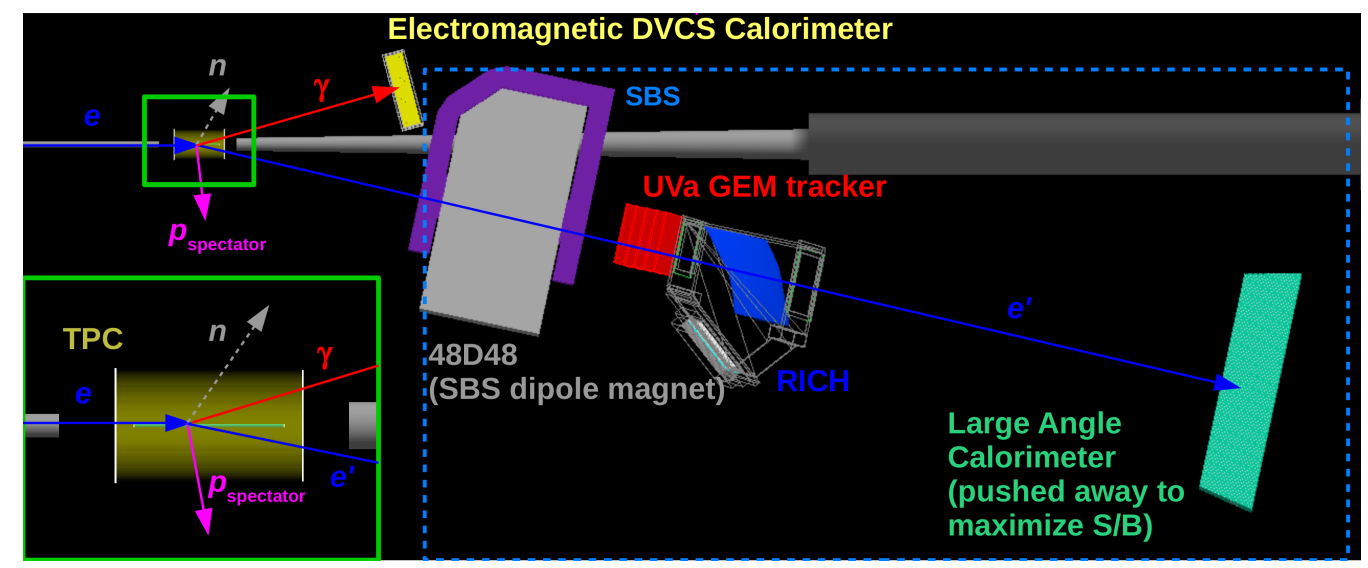

Figure 3: Geant4 model of the experimental setup for the proposed experiment.

experiment, SBS will be located at 12 degrees on the right side of the beam, and the mTPC will be located around the target. The calorimeter will be located at 14.85 degrees on the left side of the beam. We are now going to review the main instruments which will be used for this experiment.

\subsection{Experiment luminosity}

The TDIS/ $n$-DVCS target will consist of a $40 \mathrm{~cm}$ long, $1 \mathrm{~cm}$ diameter straw filled with gaseous deuterium (or hydrogen), at room temperature and $\sim 4 \mathrm{~atm}$, for a density of $6.4 \times 10^{-4} \mathrm{~g} / \mathrm{cm}^{-3}$. Combined with the beam intensity, this will provide us an instant luminosity per nucleon of $3.0 \times$ $10^{36} \mathrm{~cm}^{-2} \mathrm{~s}^{-1}$.

\subsection{Super Big Bite Spectrometer in TDIS $/ n$-DVCS setup}

The Super BigBite Spectrometer (SBS) is a $\sim 30$ msr acceptance spectrometer, composed of a single dipole magnet and a modular detector package. It has been mainly (but not exclusively) dedicated to Form Factors measurements at high $Q^{2}([8,9,10])$, for which it will serve as an hadron detector. $n$-DVCS (and TDIS), will be one of the very few SBS experiments using it in "electron mode", which requires the following detector package:

- 5 planes of GEM trackers, each made of four modules of $60 \times 50 \mathrm{~cm}^{2}$, arranged to form $60 \times 200 \mathrm{~cm}^{2}$ trackers. The hit position resolution for such trackers is projected to be below $0.1 \mathrm{~mm}$, for an angular resolution better than $1 \mathrm{mrad}$.

- the former RICH detector from HERMES [11] (usually used for hadron identification), bearing two Cherenkov radiators: an array of aerogel tiles $(n=1.03)$ covering the RICH aperture, 
and a heavy radiator gas $\left(\mathrm{C}_{4} \mathrm{~F}_{10}, n=1.00137\right)$. For our purpose (electron/pion separation), the aerogel tiles will be removed.

- one module of the CLAS Large Angle Calorimeter [12] for trigger and electron energy measurement (Fig 4 (b)). The LAC module has a rectangular shape with a sensitive area of 217 x $400 \mathrm{~cm}^{2}$ and consists of 33 layers, each composed of a $0.20 \mathrm{~cm}$ thick lead foil and $1.5 \mathrm{~cm}$ thick NE110A plastic scintillator bars.

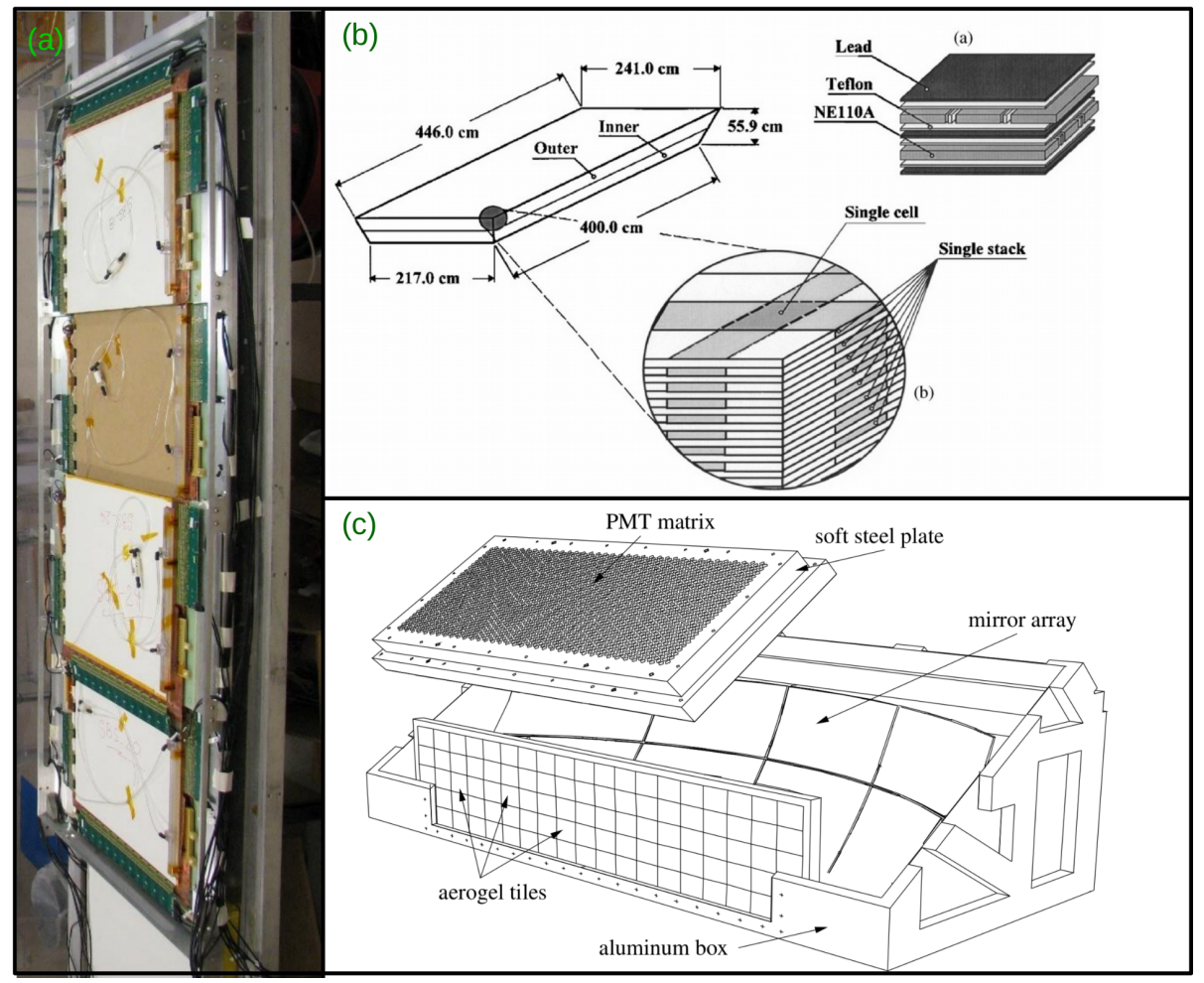

Figure 4: (a): A module of GEM tracker as it will be used in SBS;

(b): conceptual drawing of the internal structure of the LAC module;

(c): the HERMES RICH detector [11]. In electron mode, the aerogel tiles will be removed, and the gas could be substituted for lighter gas.

\subsection{1 mTPC concept}

The mTPC (for "multiple/modular Time Projection Chamber") is a $55 \mathrm{~cm}$ long time projection chamber with a cylindrical geometry, centered on and surrounding the $40 \mathrm{~cm}$ target. Its purpose is the detection of the low energy spectator protons for the TDIS measurements, or the spectator proton in $n$-DVCS on deuterium, all being within the range of 50 to 350-400 MeV/c. Fig 5 shows a drawing of this detector.

Its main feature is its longitudinal segmentation, which is capital provided the experiment luminosity and background (evaluated to be as high as $670 \mathrm{MHz}$ ). Dividing the detector not only reduces significantly the background received in each segment, but it also allows to minimize the 


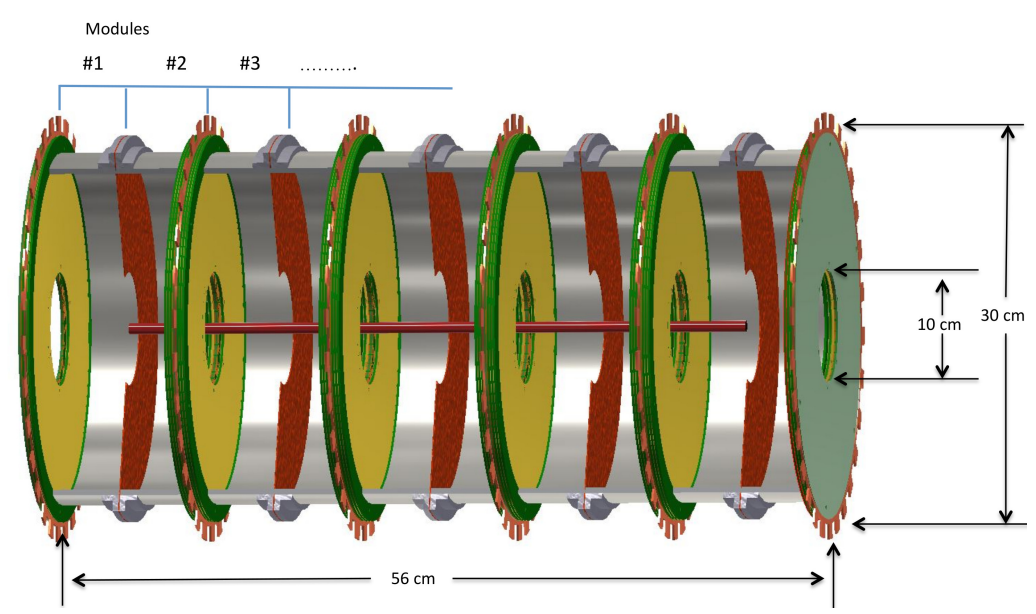

Figure 5: mTPC current design. It is composed of 10 sub-modules of $5 \mathrm{~cm}$ each, to minimize the drift time in each module and allow for a faster detector response. The yellow disks are the readout planes.

drift time down to the order of 1-2 $\mu$ s (reduction by a factor 20-40 with respect to other designs). For more techincal details about the MTPC, one may refer yet again to to the TDIS proposal [7].

The proposed modular design allows the MTPC to handle high background rates, at the cost of reduced efficiency and reduced momentum resolution due to the presence of readout disks in the middle of the active area which reduce the track lengths in the drift volume. Any track reconstructed in the mTPC with a momentum uncertainty of over $10 \%$ is rejected, leading to decreased efficiency. This is our current definition of mTPC efficiency for both the TDIS and the $n$-DVCS experiment. The efficiency has been evaluated in the range of $50 \%$ to $70 \%$ for detecting proton tracks with momenta in the range of $50 \mathrm{MeV} / \mathrm{c}$ to $300 \mathrm{MeV} / \mathrm{c}$ with a transverse momentum resolution of $10 \%$ or better. These available inefficiencies are sufficient to meet the physics goals of the TDIS program and of the n-DVCS program. We determined with our Monte Carlo simulations of en $\rightarrow$ en $\gamma$ on deuterium that the global efficiency for the detection of $n$-DVCS events with spectator proton association is $\sim 25 \%$.

\subsection{DVCS calorimeter}

The high energy photon will be measured by a dedicated electromagnetic calorimeter. Again, this calorimeter is the only specific equipment that we require to perform the $n$-DVCS measurement in addition to the TDIS setup. Our primary option for this detector is the calorimeter from the Neutral Particle Spectrometer [13].

The basic concept for the NPS is a highly segmented electromagnetic calorimeter preceded by a compact sweeping magnet. The experiments it enables, require detection of neutral particles with energies ranging between $0.5-7.6 \mathrm{GeV}$ with good energy resolution (1-2\%), and good coordinate (2-3 mm) and angular resolution of 0.5-0.75 mrad.

The NPS will consist of an array of up to 1116 scintillating $\mathrm{PbWO}_{4}$, possibly combined with up to $208 \mathrm{PbF}_{2}$ crystals. Both types of crystals are fast $\left(\mathrm{PbWO}_{4}: 5-14 \mathrm{~ns}\right.$ and $\left.\mathrm{PbF}_{2}:<30 \mathrm{~ns}\right)$ and so suitable for the experiments, which require fast signals with short tails to minimize pile-up at high rates, e.g., timing resolution of better than 100 ns. In general, the NPS needs crystals with 
high transparency, high light yield, good timing where $90 \%$ of the light is emitted within 30-50 ns, and good radiation hardness. NPS will likely take advantage of the existing $\mathrm{PbWO}_{4}$ crystals of the high-resolution inner part of the Hybrid Electromagnetic Calorimeter (HYCAL) [14, 15] used for PRIMEX /PRIMEX-II experiments.

Our plan is to use the same $\mathrm{PbWO}_{4}$ crystals NPS will use without the sweeping magnet. The crystals would be arranged similarly to the PRIMEX calorimeter, within 31 rows and 36 columns, as shown on Fig. 6. The main drawback to this option is the potential unavailability of the NPS

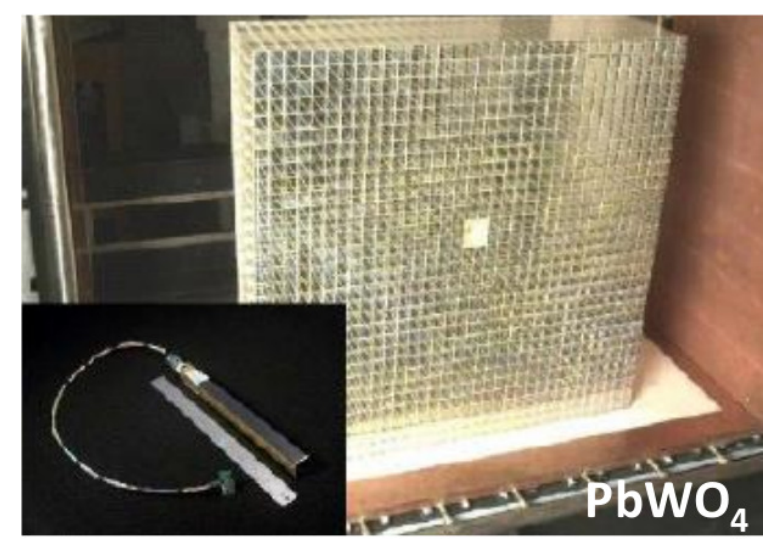

Figure 6: Inner part of the PrimEx calorimeter, composed of 1116 PbWO4 blocks. NPS will use a slightly different layout, but we plan to arrange these in a similar fashion as PRIMEX.

calorimeter $\mathrm{PbWO}_{4}$ blocks. Our backup option in this case is to use the $\mathrm{PbF}_{2}$ blocks from the Hall A DVCS calorimeter. The main disadvantage of this second option is a broader resolution both in energy (due to the lower light yield for these blocks) and in position (due to the larger transverse size of the blocks). Either way, the readout will be done using JLab FADC 250, this allows to continuously send the calorimeter data to a trigger module computing sums of adjacent $5 \times 5$ matrix of blocks. The calorimeter will be readout if one sum is above threshold of $1.5 \mathrm{GeV}$. To reduce the data, only FADC channels part of the cluster will be transferred.

\section{Analysis}

We propose to extract useful physics results with the extraction of $n$-DVCS beam spin asymmetry $A_{\mathrm{LU}}$ (the first subscript translating a longitudinally polarized beam, the second subscript $\mathrm{U}$ translating an unpolarized target), which writes as:

$$
A_{\mathrm{LU}}=\frac{N_{+}-N_{-}}{N_{+}+N_{-}}
$$

where $N_{+}$(resp. $N_{-}$) is the event yield for beam longitudinal helicity + (resp. -). The advantage of such a quantity is that it is insensitive to many systematic uncertainties, such as the uncertainties on the instruments efficiencies. 


\subsection{Selection of $n$-DVCS events}

We propose to detect our DVCS sample on the neutron in triple coincidence $D\left(e, e^{\prime} \gamma p_{s p e c}\right) n$, the scattered electron being detected by the SBS, the photon by the calorimeter, and the spectator proton $p_{\text {spec }}$ by the mTPC.

The requirements for the three detected particles are the following:

- The scattered electron should pass through all five GEM planes, leave a significant signal in the RICH (at least 4 photoelectrons), and deposit at least $0.5 \mathrm{GeV}$ in the LAC (which corresponds to a $1.5 \mathrm{GeV}$ electron); In addition, we request that the virtual photon-nucleon CMS energy $W$ is above $2 \mathrm{GeV}$, and that the virtual photon reconstructed from the electron is centered in the inner quarter of the calorimeter (see Fig 7).

- The photon is selected with a cluster in the DVCS calorimeter with an energy of at least 1 $\mathrm{GeV}$ and the reconstructed cluster position cannot be located in one of the peripheral, most outer blocks (see again Fig 7);

- The spectator proton is required to be detected by the TPC volume, and to be reconstructed with a resolution of $10 \%$.

- We require the event exclusivity with a selection on the missing mass squared distribution (see next subsection).
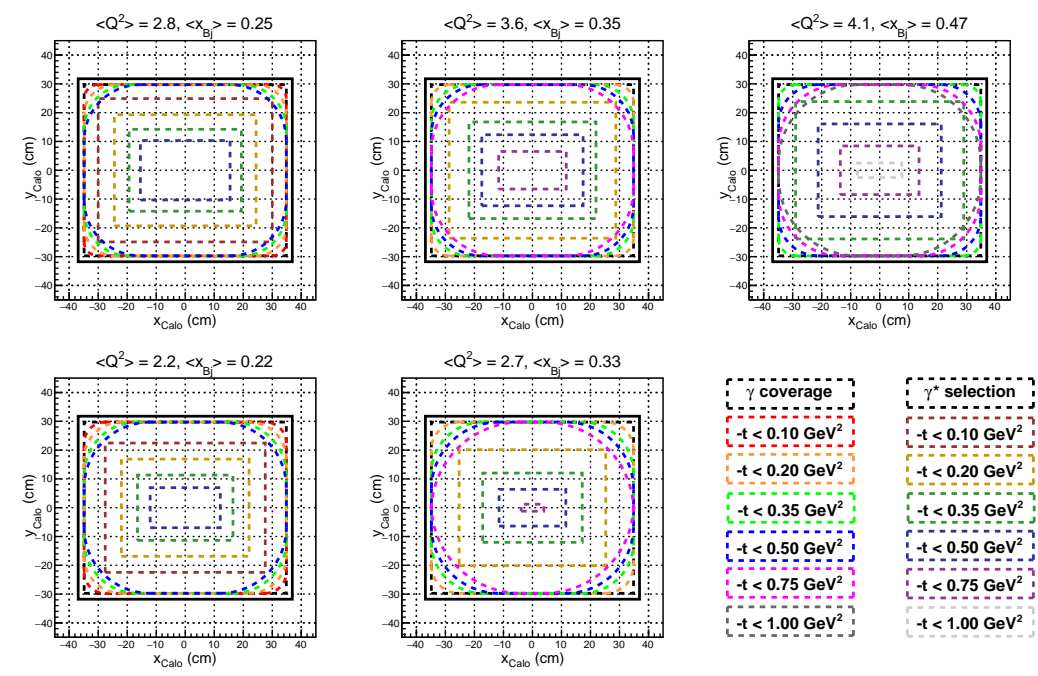

Figure 7: Selection of the virtual photon imprint and the real photon reconstructed position projected on the calorimeter surface, for all $x_{B j} / Q^{2}$ (defined in Fig 9) and $t$ bins.

\subsection{Missing mass reconstruction}

The missing mass is reconstructed with the help of the spectator proton:

$$
M_{X}^{2}=\left(k+n_{F M}-k^{\prime}-q^{\prime}\right)^{2}
$$


with $k$ and $k^{\prime}$ the 4-vectors of incident and scattered electron, $q^{\prime}$, 4-vector of produced photon, and $n_{F M}$, the 4-vector of the initial neutron inferred from the detected spectator proton $p_{\text {spec }}$ $\left(\vec{n}_{F M}=-\vec{p}_{s p e c}\right.$, within the assumption of the impulse approximation). Fig. 8 shows the effect of the inclusion of the spectator proton information on the missing mass resolution. While the spectator proton allows to reduce the missing mass width, this effect does not have the magnitude expected from the BoNuS results [16]. This means that as per our simulation, the Fermi smearing is not the driving factor of missing mass smearing, and is likely outweighed by radiative corrections at these energies. The value of the missing mass squared will provide an additional selection criterion for

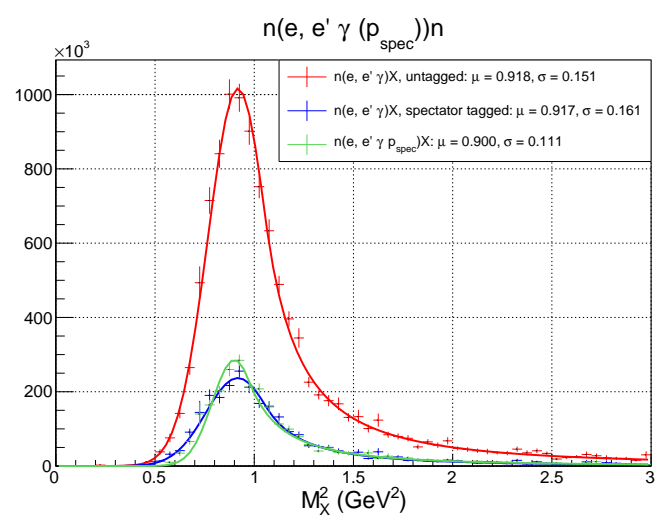

Figure 8: Effect of the detection and inclusion of the spectator proton in the reconstructed missing mass squared spectrum. Red: reconstructed missing mass squared for all $n$-DVCS events (including those not associated with a spectator proton - hence, not using its information); Blue: reconstructed missing mass squared for $n$-DVCS events with an associated spectator proton, but not using the spectator proton info in the missing mass; Green: reconstructed missing mass squared for $n$-DVCS events using the spectator proton information.

the selection of exclusive events: we require that the missing mass squared $M_{X}^{2}$ is below the pion production threshold $\left(M_{N}+m_{\pi}\right)^{2}=1.15\left(\mathrm{GeV} / \mathrm{c}^{2}\right)^{2}$. Note that in the case of the coherent DVCS on the deuterium ( $d$-DVCS), one could apply a similar selection, except we would associate the $D\left(e, e^{\prime} \gamma\right) X$ events with a recoil deuteron (which can be discriminated from spectator proton thanks to their twice as high signal amplitude/momentum ratio).

\section{Kinematical coverage}

This experiment, imagined to be combined with the TDIS experiment, will use one single setting, with SBS at 12 degrees, its front aperture $2.5 \mathrm{~m}$ from target, covering roughly $60 \mathrm{msr}$. The calorimeter will be located at 14.85 degrees, $2 \mathrm{~m}$ away from target. Fig 9 shows the kinematic coverage permitted by this setting and the proposed binning for our $n\left(e, e^{\prime} \gamma p_{\text {spec }}\right) n$ sample in the deep inelastic regime $(W>2 \mathrm{GeV}$ ). Note this binning was meant to split about evenly the statistics between each $x_{B j}, Q^{2}$ bin, and is certainly susceptible to evolve. 


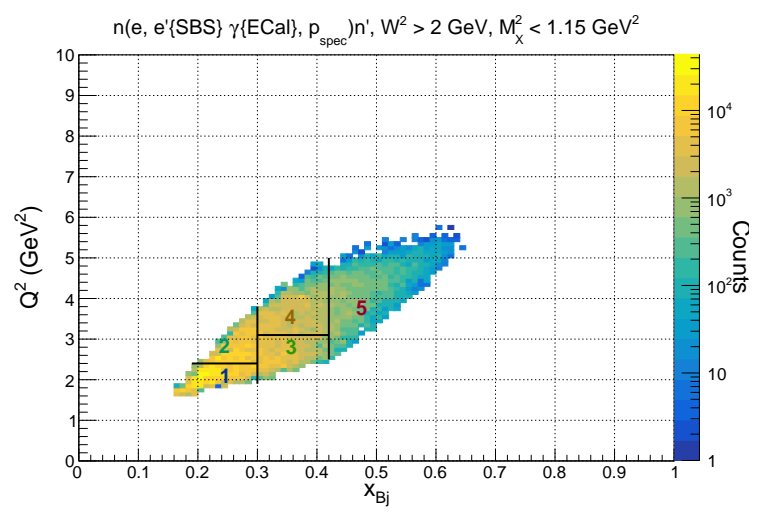

Figure 9: $Q^{2}$ vs $x_{B j}$ kinematic coverage of our $n\left(e, e^{\prime} \gamma p_{s p e c}\right) n$ sample in the deep inelastic regime $(W>2$ $\mathrm{GeV}$ ), including selection on electron, virtual and real photon, and association with spectator proton coverage.
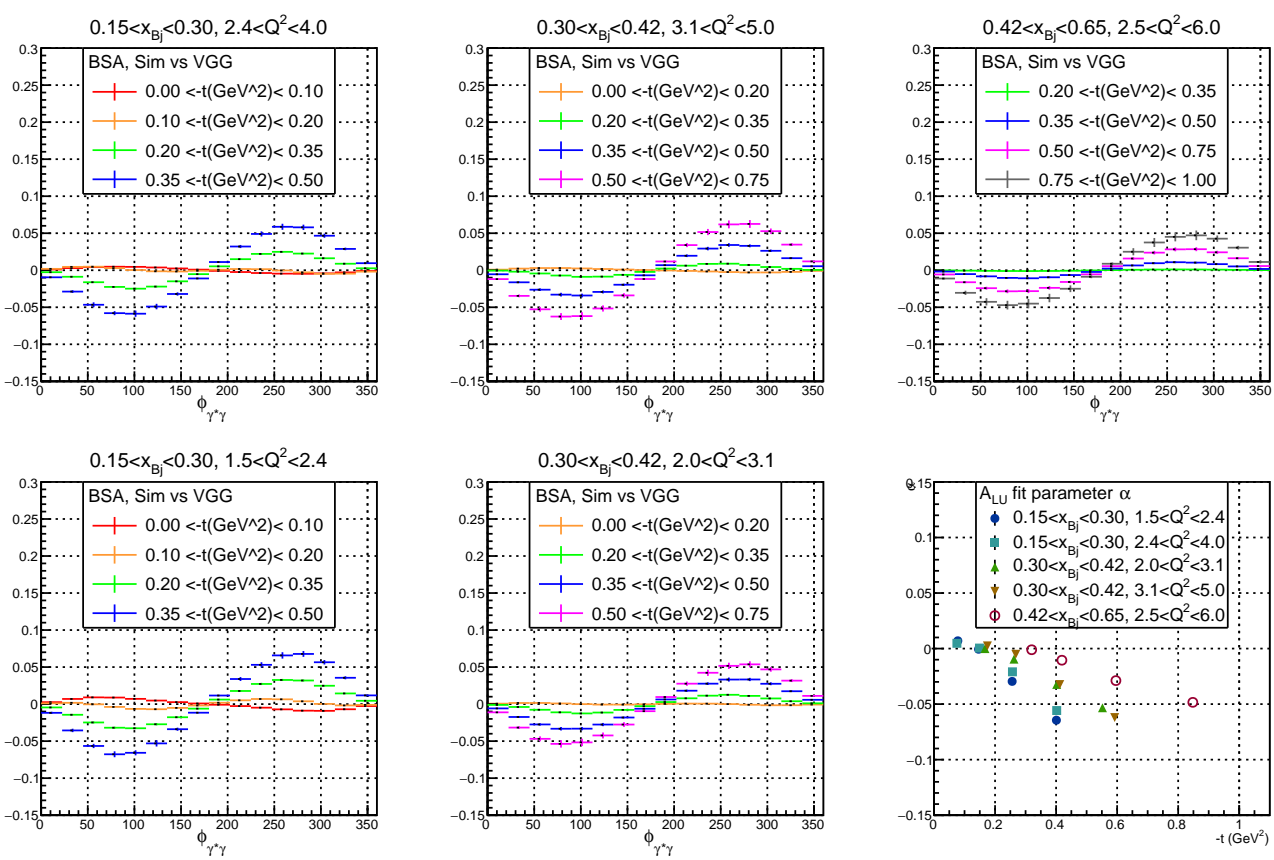

Figure 10: Projected DVCS beam spin asymmetry for the neutron as a function of $\phi_{\gamma \gamma}$ and fitted $\sin \phi_{\gamma \gamma}$ component $\alpha$ of $A_{\mathrm{LU}}$ as a function of $t$ (bottom right panel), for all kinematics defined in Fig 9.

\section{Projected results}

\subsection{Projected beam spin asymmetries}

In order to compute the projected exclusive photon electroproduction rates on the neutron (and the proton), we used a Monte Carlo simulation including the response of detectors in GEANT4, weighted with the VGG program [17] including the Bethe Heitler and the DVCS cross sections; these yields are then normalized with the experiment luminosity of $1.5 \times 10^{36} \mathrm{~cm}^{-2} \mathrm{~s}^{-1} \mathrm{n}^{-1}$. Our total expected $e n \rightarrow e n \gamma$ statistics statistics is roughly about $7 \times 10^{5}$ events. 
We have also projected the beam spin asymmetries as a function of $\phi_{\gamma \gamma}$ for the neutron for all our $x_{B j}, Q^{2}$ bins in Fig 10 . The $n$-DVCS beam spin asymmetries (estimated with VGG) are modest at low $t$ but become somewhat sizable at higher $t$ (up to $\geq 0.1$ at lower $x_{B j}, Q^{2}$, or $\geq 0.05$ at higher $x_{B j}, Q^{2}$ ), which provides us confidence in the feasibility of such a measurement.

\subsection{Comparison with ALERT}

ALERT is proposing a similar measurement to ours, in Hall B, with the CLAS12 spectrometer combined with a radial drift chamber [6]. This feature will allow them to cover a large kinematic coverage. Nonetheless, the maximal beam intensity in Hall B is intrinsically limited to $\sim 200 \mathrm{nA}$, hence their luminosity will be limited to $<10^{35} \mathrm{~cm}^{-2} \mathrm{~s}^{-1}$ ), which is an order of magnitude lower than ours. A comparison between our projected error bars for $2<Q^{2}<3 \mathrm{GeV}^{2}$ and $0.25<x_{B j}<$ 0.35 (which is one of their bins) is available on Fig. 11. This comparison highlights the statistical

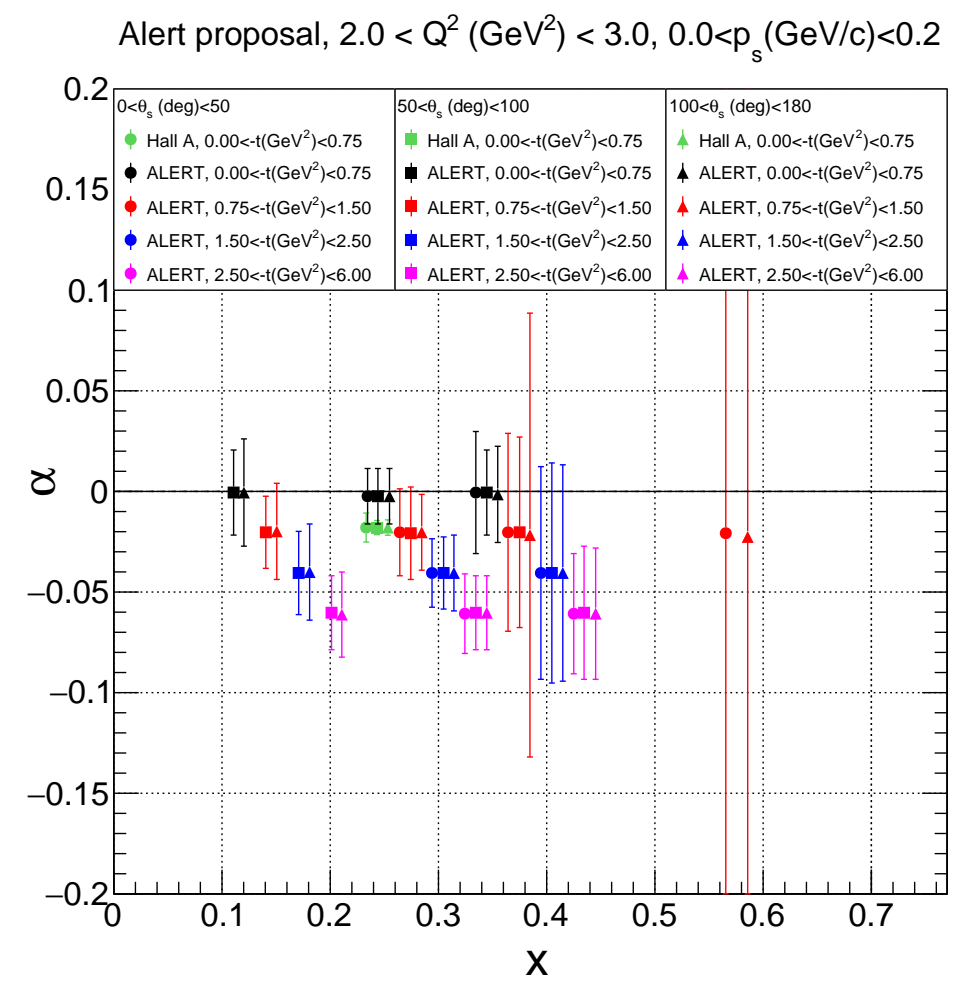

Figure 11: Projected DVCS beam spin asymmetry error bar for our experiment (green points) compared to these of ALERT (black, red, blue, and magenta points) at their $2<Q^{2}<3 \mathrm{GeV}^{2}$ kinematic point, for different values of spectator proton angle $\theta_{s}$.

advantage of our experiment, and while we are limited in kinematic coverage compared to ALERT, it has to be kept in mind that our points only represent a subset of our statistics.

\section{Summary}

The neutron DVCS experiments provide a particularly important insight of the partonic structure of the nucleon. The determination of the different contributions of $n$-DVCS observables will 
allow to access combinations of GPD integrals which are complementary to the ones provided by proton experiments. We propose an experiment which offers the possibility to mesure such a process with a reduction of the uncertainty on the identification of the spectator proton w.r.t previous similar measurements, which will bring an enhanced knowledge of the measured quantities of interest linked to the partonic structure of the nucleon.

In addition to this, this experiment may allow to measure other processes such as coherent $d$-DVCS (which can reveal new nuclear effects and can be linked to nucleon GPDs), or hard exclusive $\pi^{0}$ production off the neutron $e n \rightarrow e n \pi^{0}$, which can in principle allow access to yet other combinations of GPDs, and flavor separation. Hence, this proposal shall be completed with an estimation of the statistics expected for coherent $d$-DVCS process and hard exclusive $\pi^{0}$ production off the neutron, as well as improved estimation of systematics.

\section{References}

[1] D. Mueller, D. Robaschik, B. Geyer, F. M. Dittes, and J. Horejsi, Fortschr. Phys. 42, 101 (1994), hep-ph/9812448.

[2] A. V. Radyushkin, Phys. Rev. D56, 5524 (1997), hep-ph/9704207.

[3] X.-D. Ji, Phys. Rev. Lett. 78, 610 (1997), hep-ph/9603249.

[4] Jefferson Lab Hall A, M. Mazouz et al., Phys. Rev. Lett. 99, 242501 (2007), 0709.0450.

[5] S. Niccolai, V. Kubarovsky, A. El Alaoui, M. Mirazita (spokespersons), Deeply Virtual Compton Scattering on the Neutron with CLAS12 at $11 \mathrm{GeV}$ (2011).

[6] W. Armstrong, K. Hadifi, R. Dupré, Z.-E. Meziani (spokespersons), Spectator-Tagged Deeply Virtual Compton Scattering on Light Nuclei (2017), nucl-ex/1708.00835v1.

[7] C. Keppel, B. Wojtsekowski, P. King, D. Dutta, J.R.M. Annand, J. Zhang (spokespersons), Measurement of Tagged Deep Inelastic Scattering (TDIS): PR12-14-010 JLab experiment proposal (2014).

[8] B. Quinn, B. Wojtsekowski, R. Gilman (spokespersons), Precision Measurement of the Neutron Magnetic Form Factor up to $Q^{2}=18.0(\mathrm{GeV} / \mathrm{c})^{2}$ by the Ratio Method (2009).

[9] B. Wojtsekowski, G. Cates, S. Riordan (spokespersons), Measurement of the Neutron Electromagnetic Form Factor Ratio $G_{E}^{n} / G_{M}^{n}$ at High $Q^{2}$ (2009).

[10] C. Perdrisat, L. Pentchev, E. Cisbani, V. Punjabi, B. Wojtsekowski (spokespersons), Large acceptance Proton Form Factor Ratio measurements at 13 and $15(\mathrm{GeV} / \mathrm{c})^{2}$ using Recoil Polarization Method (2007).

[11] N. Akopov, Nucl. Instrum. Meth. A479, 511 (2002), physics/0104033.

[12] B. A. Mecking and others, Nucl. Inst. and Meth. 503, 513 (2003), nucl-ex/0711.4805.

[13] T. Horn, J. Phys.: Conf. Ser. 587, 012048 (2014), nucl-ex/1708.00835v1.

[14] PrimEx, M. Kubantsev, I. Larin, and A. Gasparian, AIP Conf. Proc. 867, 51 (2006), physics/0609201.

[15] PrimEx, A. Gasparian, A high performance hybrid electromagnetic calorimeter at Jefferson Lab, in Calorimetry in particle physics. Proceedings, 11th International Conference, CALOR 2004, Perugia, Italy, March 29-April 2, 2004, pp. 109-115, 2004. 
[16] S. Tkachenko and others, Phys. Rev. C 89, 045206 (2014).

[17] M. Vanderhaeghen, P. A. M. Guichon, and M. Guidal, Phys. Rev. D60, 094017 (1999), hep-ph/9905372. 